\title{
Preparation and Characterization of Polyurethane/Nanocopper Composites and Their Application in Intrauterine Devices
}

\author{
Yongjun Chen, Yuanfang Luo, Zhixin Jia, Demin Jia, and Jue Wang \\ Department of Polymer Materials and Engineering, South China University of Technology, \\ Guangzhou 510640, China \\ Correspondence should be addressed to Yongjun Chen; psyjchen@126.com and Zhixin Jia; zxjia@scut.edu.cn
}

Received 19 July 2013; Revised 17 September 2013; Accepted 18 September 2013

Academic Editor: Zhongkui Hong

Copyright (c) 2013 Yongjun Chen et al. This is an open access article distributed under the Creative Commons Attribution License, which permits unrestricted use, distribution, and reproduction in any medium, provided the original work is properly cited.

A novel intrauterine devices material, polyurethane/nano-copper (PU/NC) nanocomposite, was prepared. The structure, morphology, copper ion $\left(\mathrm{Cu}^{2+}\right)$ release rate, and water absorption of PU/NC nanocomposites were investigated. The results indicated that the nanocoppers were uniformly dispersed in the matrix. The release rates of $\mathrm{Cu}^{2+}$ of $\mathrm{PU} / \mathrm{NC}$ nanocomposites remained stable during the experimentation time. These results indicated that the PU/NC nanocomposites have a great potential to replace current commercial intrauterine devices materials.

\section{Introduction}

The intrauterine devices (IUDs) are the most widely used reversible method of contraception in the world today $[1,2]$. Use of IUD which can release $\mathrm{Cu}^{2+}$ in vivo is one of the effective and easy contraceptive methods. Postpartum IUD insertion, however, may increase the risk of problems, such as perforation, pain, and bleeding, which were induced by the exposed copper and the burst release of $\mathrm{Cu}^{2+}[3,4]$ causing cytotoxicity. In order to decrease the limitations of conventional $\mathrm{Cu}$-IUD materials, polymer matrix composites have been developed because of their superiority of controlled release of $\mathrm{Cu}^{2+}$ [5-8]. Polyurethanes (PUs) have been widely used for numerous biomedical applications due to their excellent mechanical properties and biocompatibility [9-12]. In this paper, new PU/NC nanocomposites were prepared in situ composite method. In this paper, polyurethane was chosen as the matrix and nanocopper was used as functional filler. The structure, morphology, $\mathrm{Cu}^{2+}$ release rate, and water absorption of PU/NC nanocomposites were investigated. PU/NC nanocomposite showed stable $\mathrm{Cu}^{2+}$ release behavior by a combination of nanostructure and hydrophilicity modification.

\section{Materials and Methods}

2.1. Materials and Instruments. Nanocopper was obtained from Shanghai Super Wei Nami Technology Co. Ltd., China; the particle size of nano-copper was $50 \mathrm{~nm}$. Diphenylmethane diisocyanate (MDI) was obtained from Yantai Wanhua Polyurethanes Co. Ltd., China. Polyethylene glycol $(\mathrm{PEG})(\mathrm{Mn}=1000)$ was obtained from Dow Chemical, USA. Polytetramethylene ether glycol (PTMG) was obtained from Mitsubishi Chemical Co., Japan. Both PEG and PTMG were dried under vacuum for $72 \mathrm{~h}$ before use. Calcium chloride dihydrate $\left(\mathrm{CaCl}_{2}\right), 1,4$-butanediol, glucose, sodium bicarbonate $\left(\mathrm{NaHCO}_{3}\right)$, and sodium phosphate monobasic dihydrate $\left(\mathrm{NaH}_{2} \mathrm{PO}_{4} \cdot 2 \mathrm{H}_{2} \mathrm{O}\right)$ were obtained from Tianjin $\mathrm{Fu}$ Chen Chemical Reagent Factory, China, and used without further purification. Commercial devices (T220c), were obtained from Yantai Family Planning Medicine \& Apparatus Co., Ltd., China. UV/Vis absorbance was measured on a UV-4802 UV/Vis Spectrophotometer (Unico Shanghai Instruments Co., Ltd., China). The morphology of the fracture surfaces of the tensile specimens was observed on a scanning electron microscope (SEM) FEI Nova Nano SEM 430 (FEI America Inc.) at an accelerating voltage of $5.0 \mathrm{kV}$. Fracture surfaces of specimen were sputter-coated with gold prior to their 
TABLE 1: Composition, contact angle, and water sorption of PU/NC nanocomposites.

\begin{tabular}{lccccccc}
\hline Samples & PEG & PTMG & MDI & BDO & Copper & Contact angle $\left(^{\circ}\right)$ & Water sorption $(\%)$ \\
\hline PU & 40 & 60 & 66.7 & 6.84 & 0 & $88.3 \pm 0.5$ & $3.7 \pm 0.2$ \\
P0 & 0 & 100 & 66.7 & 6.84 & 5 & $89.9 \pm 0.4$ & $1.1 \pm 0.1$ \\
P10 & 10 & 90 & 66.7 & 6.84 & 5 & $84.5 \pm 0.7$ & $2.7 \pm 0.1$ \\
P20 & 20 & 80 & 66.7 & 6.84 & 5 & $80.1 \pm 0.8$ & $5.0 \pm 0.1$ \\
P30 & 30 & 70 & 66.7 & 6.84 & 5 & $79.5 \pm 0.5$ & $5.4 \pm 0.1$ \\
P40 & 40 & 60 & 66.7 & 6.84 & 5 & $77.7 \pm 1.1$ & $5.8 \pm 0.2$ \\
\hline
\end{tabular}

TABLE 2: Composition of simulated uterine solution.

\begin{tabular}{lccccc}
\hline \multicolumn{7}{c}{ Concentration in water $(\mathrm{g} / \mathrm{L})$} \\
$\mathrm{NaHCO}_{3}$ & $\mathrm{NaH}_{2} \mathrm{PO}_{4} \cdot 2 \mathrm{H}_{2} \mathrm{O}$ & Glucose & $\mathrm{CaCl}_{2}$ & $\mathrm{KCl}$ & $\mathrm{NaCl}$ \\
\hline 0.25 & 0.072 & 0.50 & 0.167 & 0.224 & 4.97 \\
\hline
\end{tabular}

observation. The contact angles of the composites were measured on a Drop Shape Analysis System DSA100 (Krüss $\mathrm{GmbH}$, Germany). ATR-FTIR analysis was conducted using a VERTEX 70 FT-IR (Bruker Optics, Inc., Germany) with an ATR accessory.

2.2. Preparation of PU/Nanocopper Composites. The compositions of the $\mathrm{PU} /$ nano-copper composites were show in Table 1. The PEG and PTMG were first mixed at $80^{\circ} \mathrm{C}$ for $1 \mathrm{~h}$, and then the NC particles were dispersed in the mixture of PEG and PTMG via an ultrasonicator for $1 \mathrm{~h}$ at $80^{\circ} \mathrm{C}$. Then, the stoichiometric amount of MDI was added to the suspension and reacted at $80^{\circ} \mathrm{C}$ for $2 \mathrm{~h}$, yielding the prepolyurethane. The whole reaction was carried out under nitrogen with mechanical stirring. The stoichiometric amount of 1,4-butanediol (it was calculated according to the residual amount of $\mathrm{NCO}$ ) was mixed with the prepolyurethane for $60 \mathrm{~s}$ under a violent stirring condition at $110^{\circ} \mathrm{C}$. Subsequently, the mixture was cured at $100^{\circ} \mathrm{C}$ under pressure for $10 \mathrm{~h}$ in a metal mould.

2.3. Measurement of $\mathrm{Cu}^{2+}$ Release Rate in Simulated Uterine Solutions. Absorbance measurements were employed to measure the $\mathrm{Cu}^{2+}$ release rate of PU/NC nanocomposites and $\mathrm{T} 220 \mathrm{c}$ in a simulated uterine solution according to the previous literature [13-15]. The composition of the simulated uterine solution is shown in Table 2. The $\mathrm{pH}$ value of 6.3 was established by adding dilute hydrochloric acid or sodium hydroxide solution and was adjusted periodically throughout the exposure time. Three specimens of every composite were prepared with a size of $5.0 \times 5.0 \times 1.0 \mathrm{~cm}$ and then suspended into $50 \mathrm{~mL}$ simulated uterine solution at $37.0 \pm 0.1^{\circ} \mathrm{C}$. The amount of released $\mathrm{Cu}^{2+}$ of PU/NC nanocomposites and the commercial IUD was determined on the UV-4802 weekly for 3 months.

\section{Results and Discussion}

3.1. Structure Characterization of PU/NC Nanocomposites. The ATR-FTIR spectroscopy of PU and the P40 are shown in Figure 1. The $\mathrm{N}-\mathrm{H}$ stretching vibrations bonds were detected at $3150-3500 \mathrm{~cm}^{-1}$. The peaks at 2935 and $2855 \mathrm{~cm}^{-1}$

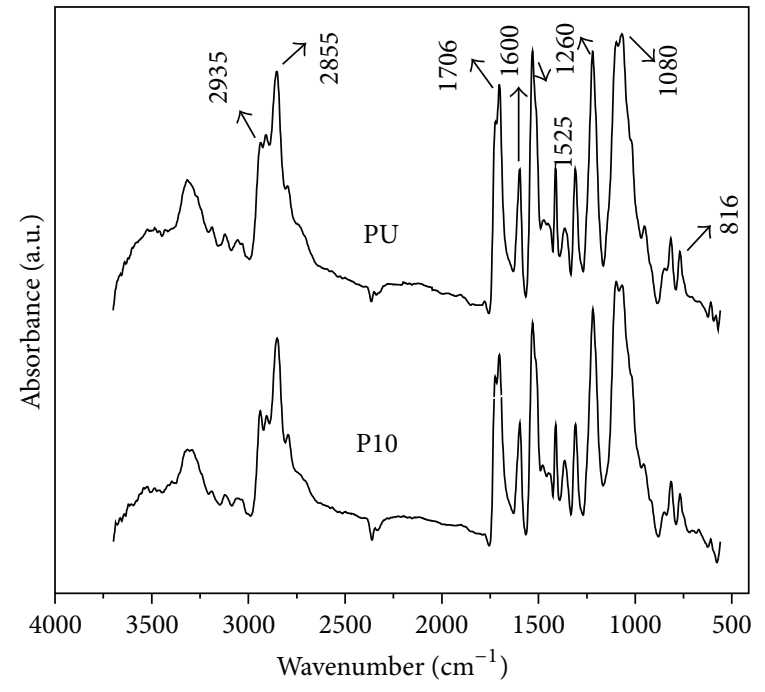

FIGURE 1: FT-IR spectra of PU and P10.

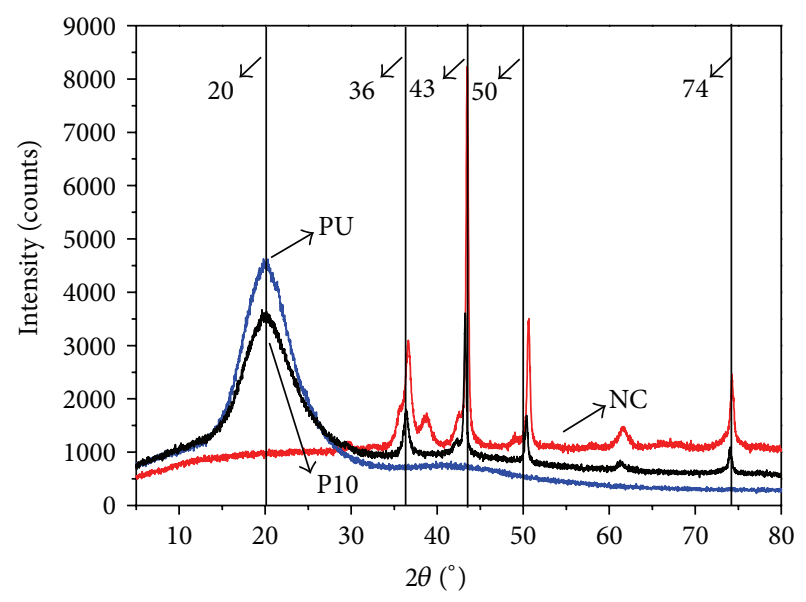

FIGURE 2: XRD patterns of PU, nano-copper and P10.

were attributed to $\mathrm{CH}_{2}$ asymmetric and symmetric stretching vibrations, respectively. The peaks between 1600 and $1800 \mathrm{~cm}^{-1}$ indicated the $\mathrm{C}-\mathrm{O}$ stretching vibrations, and the $1520-1550 \mathrm{~cm}^{-1}$ are associated with the urethane N-H bending $\mathrm{C}-\mathrm{N}$ stretching. The typical peaks of PEG and PTMG were also detected at $1260 \mathrm{~cm}^{-1}$ (symmetric $\mathrm{CH}_{3}$ bending), $1080 \mathrm{~cm}^{-1}$ (C-O-C stretching), and $816 \mathrm{~cm}^{-1}\left(\mathrm{CH}_{3}\right.$ rocking), respectively [16]. 
TABLE 3: Average $\mathrm{Cu}^{2+}$ release rates of PU/NC nanocomposites for the first month.

\begin{tabular}{lccccc}
\hline Samples & P0 & P10 & P20 & P30 & P40 \\
\hline $\mathrm{Cu}^{2+}$ release rates $(\mu \mathrm{g} /$ day $)$ & $42.2 \pm 1.2$ & $46.5 \pm 1.1$ & $45.0 \pm 1.8$ & $38.7 \pm 1.1$ & $60.4 \pm 1.6$ \\
\hline
\end{tabular}

TABLE 4: $\mathrm{Cu}^{2+}$ release rates of PU/NC nanocomposites at the end of three months.

\begin{tabular}{lccccc}
\hline Samples & P0 & P10 & P20 & P30 & P40 \\
\hline $\mathrm{Cu}^{2+}$ release rates $(\mu \mathrm{g} /$ day $)$ & $33.1 \pm 1.0$ & $46.0 \pm 1.1$ & $52.4 \pm 1.2$ & $65.9 \pm 1.3$ & $73.7 \pm 1.7$ \\
\hline
\end{tabular}

3.2. X-Ray Diffraction Analysis. Figure 2 shows the XRD patterns of PU, nano-copper and P40. PU had broad diffraction peaks in $20^{\circ}$ and $42^{\circ}$ because there were no microcrystalites in PU samples. In the XRD spectra of the PU/NC nanocomposite, the peaks at $36^{\circ}$ belonged to cuprous oxide, and the peaks in $43^{\circ}, 50^{\circ}$, and $74^{\circ}$ belonged to the nano-copper.

3.3. Contact Angle and Water Sorption. Contact angle and water sorption of the PU/nanocopper, nanocomposites are summarized in Table 1. In this research, the hydrophilicity of the nanocomposites increased with the increasing content of the PEG. The contact angle and water sorption of the PU/NC nanocomposites increased with increasing the content of PEG in the soft segments of the composites. This is because the water sorption of the nanocomposites was influenced by the hydrophilicity of the nanocomposites $[17,18]$. Therefore, the contact angle and water sorption of nanocomposites increased with increasing the content of the hydrophilic segments, for PEG is more hydrophilic than PTMG.

3.4. $\mathrm{Cu}^{2+}$ Release Rate of the PU/NC Nanocomposite. The $\mathrm{Cu}^{2+}$ release rates of the composites are shown in Figure 3. The average $\mathrm{Cu}^{2+}$ release rates of P0, P10, P20, P30, and P40 are $42.4,46.5,45.0$, and $60.4 \mu \mathrm{g} / \mathrm{day}$, respectively. In the first month (as shown in Table 3), there were no burst releases because the nano-copper was wrapped in the polyurethane segments. However, the $\mathrm{Cu}^{2+}$ release rates of the PU/NC nanocomposites increased with increase the content of PEG in the soft segment. It may be attributes to the hydrophilicity of the composites. As shown in Figure 3, after six weeks, the release rates of the $\mathrm{PU} / \mathrm{NC}$ nanocomposites remained relatively stable after six weeks.

The relative $\mathrm{Cu}^{2+}$ release rates of $\mathrm{P} 10$ and commercial $\mathrm{T} 220 \mathrm{c}$ are shown in Figure 4 . The $\mathrm{Cu}^{2+}$ release rate of T220c showed a burst release in the first two weeks, and the average release rate was about $94 \mu \mathrm{g} /$ day in the first three months. But the average $\mathrm{Cu}^{2+}$ release rate per day in the first three months of P10 was only about $46 \mu \mathrm{g} / \mathrm{day}$, and there was no initial burst (as shown in Table 4). The average $\mathrm{Cu}^{2+}$ release rate was always about 35-55 $\mu \mathrm{g} /$ day. This indicates that the PU/NC nanocomposites have a great potential to replace current commercial Cu-IUD materials.

3.5. Microstructure of the PU/NC Nanocomposites. To investigate the dispersion state of nano-copper in the polyurethane matrix, the tensile fractured surface of P10 was examined by SEM (Figure 5). A uniform dispersion of nano-copper was observed, and no obvious aggregation occurred.

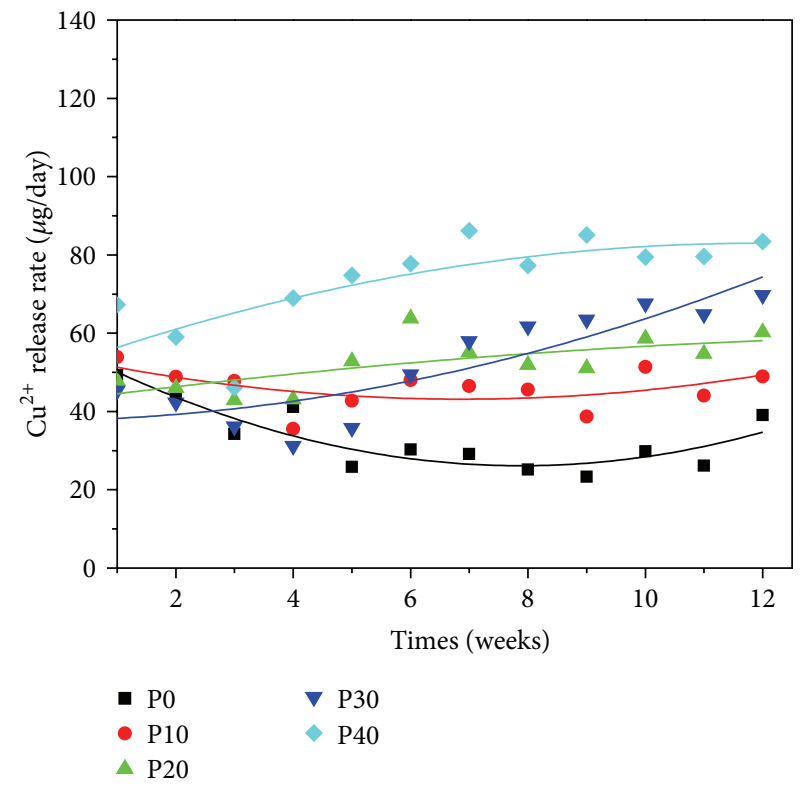

FIGURE 3: $\mathrm{Cu}^{2+}$ release rates of PU/NC nanocomposites.

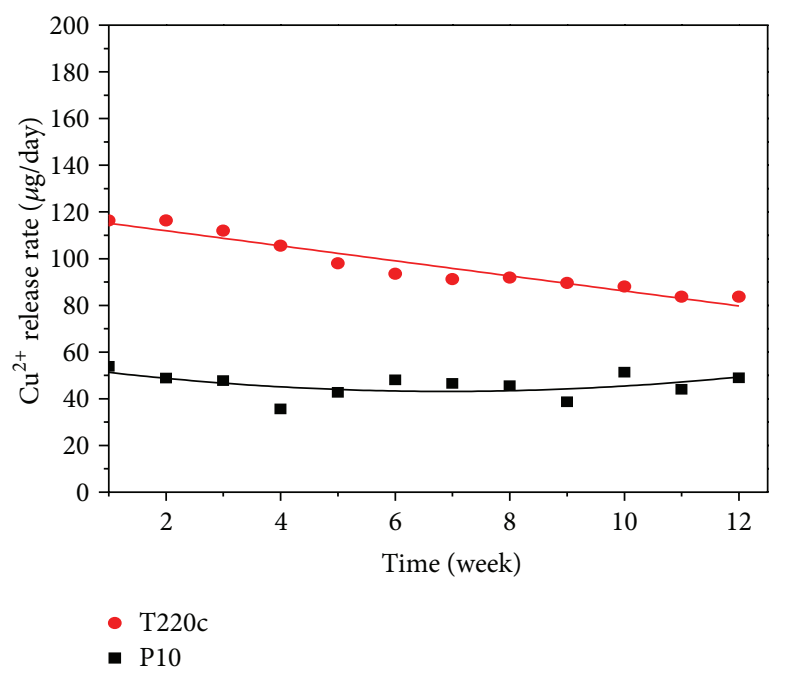

FIgURE 4: $\mathrm{Cu}^{2+}$ release rates of T220c and P10.

\section{Conclusions}

PU/NC nanocomposites were prepared and used as intrauterine devices. The nano-copper was uniformly dispersed in the nanocomposites. The burst release of the $\mathrm{Cu}^{2+}$ could 


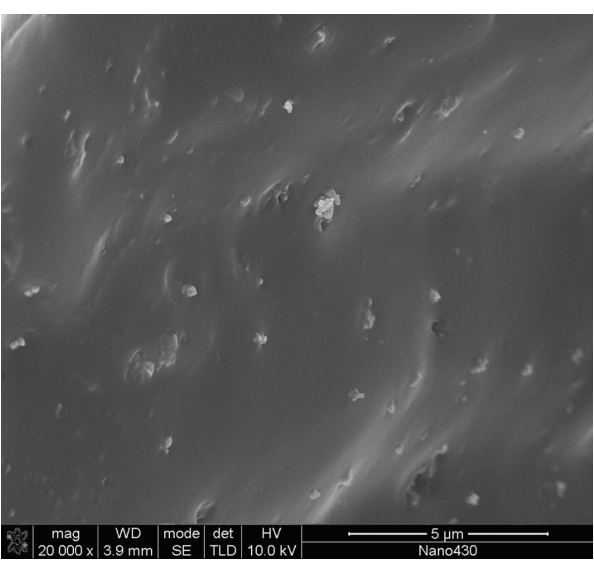

(a)

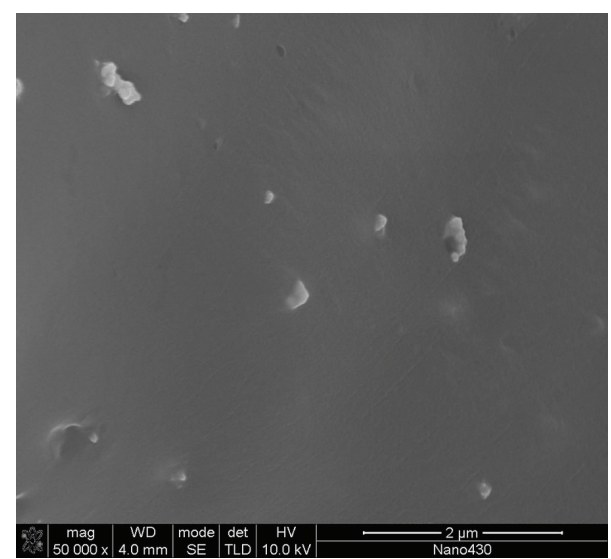

(b)

Figure 5: The SEM image of P10.

be eliminated completely, and the release rates remained relatively stable over three months. These results indicate that the PU/NC nanocomposites have a great potential to replace current commercial intrauterine devices.

\section{Conflict of Interests}

The authors declare that there is no conflict of interests regarding the publication of this paper.

\section{Acknowledgments}

The authors thank the Fundamental Research for the Joint Funds of the National Natural Science Foundation of China (U1134005), the Central Universities (2012ZM0009) for financial supports, the National Natural Science Foundation of China (51003031), and the National Key Technology R\&D Program of China (2012BAE01B03).

\section{References}

[1] M. C. Fox, J. Oat-Judge, K. Severson et al., "Immediate placement of intrauterine devices after first and second trimester pregnancy termination," Contraception, vol. 83, no. 1, pp. 34-40, 2011.

[2] R. Kulier, P. A. O'Brien, F. M. Helmerhorst, M. Usher-Patel, and C. D'Arcangues, "Copper containing, framed intra-uterine devices for contraception," Cochrane Database of Systematic Reviews, vol. 17, no. 4, 2007.

[3] L. Patchen and E. K. Berggren, "Use of the copper T380A intrauterine device by adolescent mothers: continuation and method failure," Journal of Pediatric and Adolescent Gynecology, vol. 24, no. 2, pp. 71-73, 2011.

[4] X. Xia, Y. Tang, C. Xie, Y. Wang, S. Cai, and C. Zhu, "An approach to give prospective life-span of the copper/lowdensity-polyethylene nanocomposite intrauterine device," Journal of Materials Science, vol. 22, no. 7, pp. 1773-1781, 2011.

[5] X. Xia, S. Cai, J. Hu, and C. Xie, "Water absorption characteristics of novel $\mathrm{Cu} / \mathrm{LDPE}$ nanocomposite for use in intrauterine devices," Journal of Biomedical Materials Research B, vol. 79, no. 2, pp. 345-352, 2006.
[6] J. Li, J. Suo, X. Huang, C. Ye, and X. Wu, "Release behavior of copper ion in a novel contraceptive composite," Contraception, vol. 76, no. 3, pp. 233-237, 2007.

[7] Z. Yang, C. Xie, H. Xiang, J. Feng, X. Xia, and S. Cai, "IDM release behavior and surface characteristics of the novel $\mathrm{Cu} / \mathrm{IDM} / \mathrm{LDPE}$ nanocomposite for intrauterine device," Colloids and Surfaces B, vol. 69, no. 2, pp. 276-280, 2009.

[8] X. Zhou, Y. Li, X. Jiang, L. Qiu, and J. Liu, "Release of copper and indomethacin from intrauterine devices immersed in simulated uterine fluid," European Journal of Contraception and Reproductive Health Care, vol. 15, no. 3, pp. 205-212, 2010.

[9] S. Dawlee and M. Jayabalan, "Development of segmented polyurethane elastomers with low iodine content exhibiting radiopacity and blood compatibility," Biomedical Materials, vol. 6, no. 5, Article ID 055002, 2011.

[10] P. P. Vicario, Z. Lu, Z. Wang, K. Merritt, D. Buongiovanni, and P. Chen, "Antithrombogenicity of hydromer's polymeric formula F202 immobilized on polyurethane and electropolished stainless steel," Journal of Biomedical Materials Research B, vol. 86, no. 1, pp. 136-144, 2008.

[11] L. Peng and G. R. Kinsel, "Improving the sensitivity of matrixassisted laser desorption/ionization (MALDI) mass spectrometry by using polyethylene glycol modified polyurethane MALDI target," Analytical Biochemistry, vol. 400, no. 1, pp. 56-60, 2010.

[12] H.-S. Choi, H. Suh, J.-H. Lee et al., "A polyethylene glycol grafted bi-layered polyurethane scaffold: preliminary study of a new candidate prosthesis for repair of a partial tracheal defect," European Archives of Oto-Rhino-Laryngology, vol. 265, no. 7, pp. 809-816, 2008.

[13] B. Cao, T. Xi, and Y. Zheng, "Release behavior of cupric ions for TCu380A and TCu220C IUDs," Biomedical Materials, vol. 3, no. 4, Article ID 044114, 2008.

[14] S. Cai, X. Xia, C. Zhu, and C. Xie, "Cupric ion release controlled by copper/low-density polyethylene nanocomposite in simulated uterine solution," Journal of Biomedical Materials Research $B$, vol. 80, no. 1, pp. 220-225, 2007.

[15] Y. Feng, W.-K. Teo, K.-S. Siow, K.-L. Tan, and A.-K. Hsieh, "The corrosion behaviour of copper in neutral tap water. Part I: corrosion mechanisms," Corrosion Science, vol. 38, no. 3, pp. 369-385, 1996.

[16] E. Campos, R. Cordeiro, A. C. Santos, C. Matos, and M. H. Gil, "Design and characterization of bi-soft segmented 
polyurethane microparticles for biomedical application," Colloids and Surfaces B, vol. 88, no. 1, pp. 477-482, 2011.

[17] M. Shibaya, Y. Suzuki, M. Doro, H. Ishihara, N. Yoshihara, and M. Enomoto, "Effect of soft segment component on moisturepermeable polyurethane films," Journal of Polymer Science B, vol. 44, no. 3, pp. 573-583, 2006.

[18] S. Mondal and J. L. Hu, "Structural characterization and mass transfer properties of nonporous segmented polyurethane membrane: influence of hydrophilic and carboxylic group," Journal of Membrane Science, vol. 274, no. 1-2, pp. 219-226, 2006. 

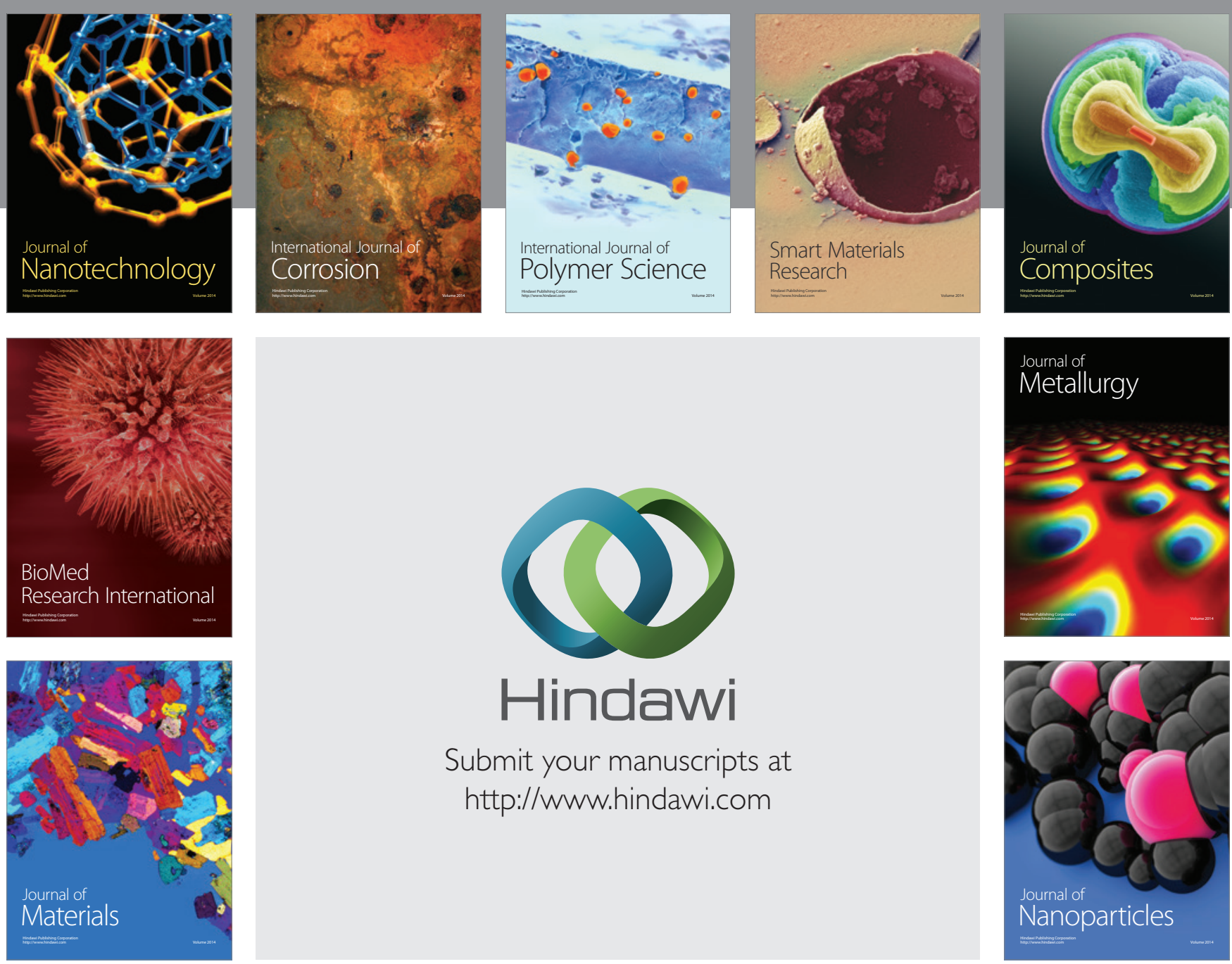

Submit your manuscripts at http://www.hindawi.com
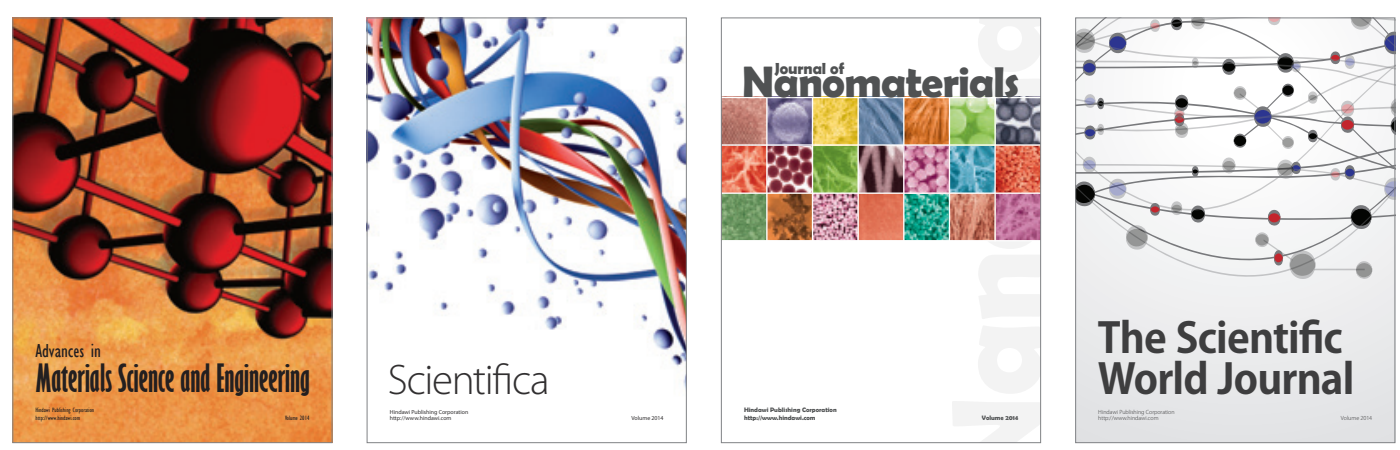

\section{The Scientific World Journal}
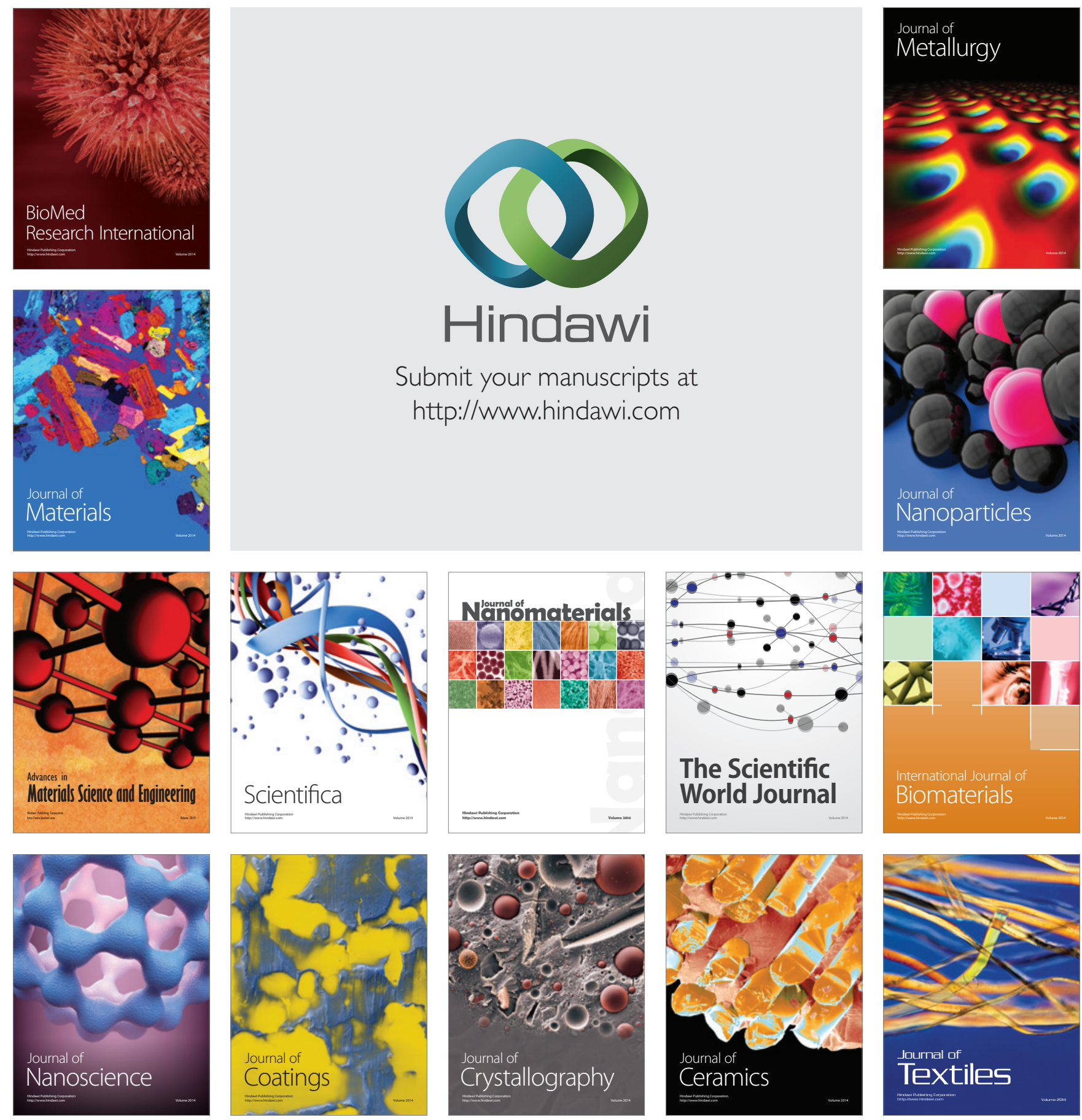